\title{
Editorial: The Role of Climate and Air Pollution in Human Health and Urban Chemistry in Asian Cities
}

\author{
Prashant Rajput ${ }^{1 *}$, Atinderpal Singh ${ }^{2}$, Jai Prakash ${ }^{3}$ and Manish Kumar ${ }^{4}$ \\ ${ }^{1}$ DST-Mahamana Centre of Excellence in Climate Change Research, IESD, Banaras Hindu University, Varanasi, India, \\ ${ }^{2}$ Department of Environmental Studies, University of Delhi, New Delhi, India, ${ }^{3}$ Energy, Environmental and Chemical \\ Engineering, Washington University in St. Louis, St. Louis, MO, United States, ${ }^{4}$ Department of Environmental Science and \\ Analytical Chemistry (ACES), Stockholm University, Stockholm, Sweden
}

Keywords: climate change in Asian cities, air pollution in Asian cities, human health, urban chemistry, aerosols, trace gases, urban hotspots, atmospheric chemistry

\section{Editorial on the Research Topic}

The Role of Climate and Air Pollution in Human Health and Urban Chemistry in Asian Cities

\section{OPEN ACCESS}

Edited and reviewed by: Prashant Kumar University of Surrey, United Kingdom

*Correspondence: Prashant Rajput prajput.pr@gmail.com

Specialty section: This article was submitted to Climate Change and Cities, a section of the journal Frontiers in Sustainable Cities

Received: 19 January 2022

Accepted: 27 January 2022

Published: 18 February 2022

Citation:

Rajput P, Singh A, Prakash J and Kumar M (2022) Editorial: The Role of

Climate and Air Pollution in Human Health and Urban Chemistry in Asian Cities. Front. Sustain. Cities 4:858060 doi: 10.3389/frsc.2022.858060
The Asian cities are experiencing unprecedented climate, poor air quality, and human health due to existing rapid urbanization, air pollution, unsustainable land-use planning, and industrialization. Therefore, air pollution is now one of the biggest threats to human health in urban cities of Asia. The association between air pollution and human health is very complex due to atmospheric processes and the transformation of pollutants which are considered as exposure variables. Furthermore, biomass burning and climate change effects for example rise in temperature and the perturbed hydrological cycle can significantly impact the atmospheric chemistry, and human health in urban air-sheds in Asia.

A total of 10 articles are provided in this special issue, which is aimed at recent developments in the area of air pollution, urban atmospheric chemistry, and its impact on public health and climate change in Asian cities. This issue is centered on the novelty of work reported from experimental as well as modeling analysis in the field of air pollution, climate change, and human health. The manuscripts went through a rigorous, transparent, single-blinded, and interactive peer-review process involving the authors, the Reviewers, and the Guest Editors prior to acceptance for publication in this special issue. The gist of these publications is given below:

Many researchers have reported the bursting of firecrackers (FC) during the Diwali festival (celebrated on a particular date either in the month of October or November) in India which leads to further increase the air pollution above the background levels and plausibly affects the human health. During post-monsoon (October-November), the long-range transport of biomass burning emissions (LRT-BB) also affects the air quality in downwind locations in Northern India. Rajput et al. revealed that FC burst in Diwali and LRT-BB increased the daily $\mathrm{PM}_{2.5}$ concentration by 11 and $36 \%$, respectively over its urban background level $\left(286 \mu \mathrm{g} \mathrm{m}^{-3}\right)$ at Kanpur using the Lenschow-type approach on a temporal domain for the first time. Bangar et al. reported the source apportionment of $\mathrm{PM}_{2.5}$ from Northern India (Delhi) during the post-monsoon season and they also found that LRT-BB has the highest contribution to $\mathrm{PM}_{2.5}$ during this season as reported by Rajput et al. Along with LRT-BB, they identified the other major sources of $\mathrm{PM}_{2.5}$ such as uplifted mineral dust, vehicular emissions, road dust resuspension, secondary aerosols formation, industrial emission, coal combustion, and solid-waste burning.

The urban population is subjected to multiple exposures to air pollution and heat stress that have several negative health impacts. Indian cities are highly vulnerable to extreme weather events for example heatwaves and cold waves. A review article by Menon and Sharma highlights the use Nature-Based Solutions (NBS) to tackle the environmental issue due to their multi-functional 
nature and cost-effectiveness. In their review article, they highlighted co-benefits of using NBS such as reduction in energy cost as well as conservation of biodiversity in addition to improving public health (through a reduction in air pollution and urban heat). Dutta et al. examined the impact of heat stress on the stone quarry workers. The result findings suggest that around $14 \%$ of workers were vulnerable to heat stress.

Tuladhar et al. estimated the health impacts of $\mathrm{PM}_{2.5}$ exposure using the WRF-Chem Model in Kathmandu Valley, Nepal. The exposure analysis indicates that 19 people could die due to lung cancer and 175 people could die due to all-cause (non-accidental) diseases due to $\mathrm{PM}_{2.5}$ exposure in December. Furthermore, their simulation estimated that reducing the $50 \% \mathrm{PM}_{2.5}$ level in the valley could lead to a reduction in the monthly mortality by $51.4 \%$. Pavel et al. estimated the human health risk due to the criteria pollutants in Dhaka, Bangladesh. They found that hazard quotient (HQ) values were not antagonistic (HQ $<1$ ) while assessing acute exposure in the three age groups (infants, children, and adults). However, their study showed a significant health risk $(\mathrm{HQ}>1)$ in chronic exposure for infants and children. They identified children are the worst sufferers among the age groups. Air pollution due to nanoparticles (NPs) is receiving increasing attention in scientific communities due to their strong influence on human health. Sonwani et al. provided a comprehensive review on the atmospheric NPs and their association with human health. Exposure to NPs causes the generation of ROS, resulting in cytotoxicity that leads to genotoxicity and tumorigenesis. The overproduction of ROS and the weakening of the antioxidant defense system cause oxidative stress which can trigger the release of more pro-inflammatory hormones that lead to inflammation as well as acute and chronic lung diseases.

Climate change is one of the biggest challenges of sustainability in today's world. Kaur and Pandey reviewed the present status of climate change, air pollution, and human health in Indian cities. In this review, they stated that the Indian population is experiencing adverse human health impacts due to air pollution and climate change. Further, they emphasized the role of climate change in arising extreme weather events in India.
They also highlighted the use of satellite data with geospatial techniques in monitoring and mapping spatial-temporal distribution patterns of air pollution and climate change and associated health impacts. Therefore, to make sustainable cities in developing countries like India, there is a need for stringent urban planning, electric mobility, and action plans to curtail urban air pollution and improve the public health system.

The COVID-19 pandemic has affected our economic growth and health care system. Mishra et al. assessed the impact of lockdown and unlock phases on ambient atmospheric air quality parameters across 16 major cities of India covering the northto-south stretch of the country. They reported a reduction in $\mathrm{PM}_{2.5}$ by $49 \%$ over north India during the lockdown period. Their results indicate that by adopting cleaner fuel technology and avoiding poor combustion activities across the urban cities of India a reduction in $\mathrm{PM}_{2.5}$ up to $30 \%$ can be achieved. Another study (Yadav et al.) focused on the substantial improvement in air quality over 6 cities of the states of Rajasthan (India) during the nationwide lockdown amid COVID-19.

\section{AUTHOR CONTRIBUTIONS}

All authors listed have made a substantial, direct, and intellectual contribution to the work and approved it for publication.

Conflict of Interest: The authors declare that the research was conducted in the absence of any commercial or financial relationships that could be construed as a potential conflict of interest.

Publisher's Note: All claims expressed in this article are solely those of the authors and do not necessarily represent those of their affiliated organizations, or those of the publisher, the editors and the reviewers. Any product that may be evaluated in this article, or claim that may be made by its manufacturer, is not guaranteed or endorsed by the publisher.

Copyright (C) 2022 Rajput, Singh, Prakash and Kumar. This is an open-access article distributed under the terms of the Creative Commons Attribution License (CC BY). The use, distribution or reproduction in other forums is permitted, provided the original author(s) and the copyright owner(s) are credited and that the original publication in this journal is cited, in accordance with accepted academic practice. No use, distribution or reproduction is permitted which does not comply with these terms. 